\title{
Association/Dissociation of Caveolin-1 and Nitric Oxide Synthase in Preserving Renal Medulla Function during Hemorrhage and Aging
}

\author{
Noelia Daniela Arreche ${ }^{1 *}$, Gustavo Cernadas ${ }^{1}$, María Bernardita Puchulu ${ }^{1,2}$, Andrea Lorena \\ Fellet $^{1,2}$ and Ana María Balaszczuk ${ }^{1,2}$ \\ ${ }^{1}$ Departamento de Ciencias Biológicas, Universidad de Buenos Aires, Argentina
}

${ }^{2}$ Facultad de Farmacia y Bioquímica, Instituto de Química y Metabolismo del Fármaco IQUIMEFA- CONICET, Universidad de Buenos Aires, Argentina

*Corresponding author: Noelia Daniela Arreche, Department of Physiology, School of Pharmacy and Biochemistry, University of Buenos Aires, C1113AAD Buenos Aires, Argentina.

To Cite This Article: Noelia Daniela A, Gustavo C, María Bernardita P, Andrea Lorena F, Ana María B. H Abdulmajed. Association/Dissociation of Caveolin-1 and Nitric Oxide Synthase in Preserving Renal Medulla Function during Hemorrhage and Aging. Am J Biomed Sci \& Res. 2021 - 11(4). AJBSR.MS.ID.001655. DOI: 10.34297/AJBSR.2021.11.001655.

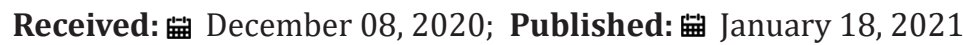

\begin{abstract}
Background: Caveolin-1 is a negative regulator of endothelial nitric oxide synthase (eNOS), as its interaction and binding suppress the activity of eNOS by making caveolin-eNOS complex. The critical role of caveolin-1 and eNOS in the renal medulla during hypovolemic state and aging is unclear.

Objective: To investigate changes in the renal medulla the activity and protein levels of the different NOS forms, changes in the abundance of caveolin-1 and caveolin-1/eNOS association in response to acute hemorrhage in young and middle-aged rats.

Methods: In the present study we determined: i) NOS activity and protein levels of the different NOS isoforms, ii) the abundance of caveolin-1 and, iii) caveolin-1/eNOS association during hypovolemic state of young and middle-aged rats in the kidney.

Results: We have demonstrated that caveolin-1 is involved in the regulation of renal NOS activity in response to acute hemorrhage and aging.

Conclusion: Our data showed that association/dissociation of caveolin-1 and eNOS in renal medulla play a critical role in preserving the renal function during hypovolemic state in young and middle-aged rats.
\end{abstract}

Keywords: Caveolin-1; Nitric oxide; Hemorrhage; Aging; Kidney

\section{Introduction}

Many organfailures are a common consequence of a hypovolemic disorder, the kidney being one of the primary target organs [1]. It was reported that one of the most important physiological systems involved in hydrosaline homeostasis is nitric oxide (NO) pathway [2]. We recently demonstrated an involvement of NO and caveolins in the age-associated functional and structural changes in a heart under osmotic stress caused by a long-term liquid restriction [3]. Like the cardiac system, NO also plays a significant role during renal aging process [4]. Aging kidneys undergo structural and functional changes decreased autoregulatory capacity and increase susceptibility to acute stress [5]. There is a substantial literature indicating a progressive endothelial dysfunction with aging, which includes a reduction in NO-dependent responses [6,7]. This reduced peripheral NO activity could reflect a fall in NO synthesis, perhaps secondary to lack of NOS or essential cofactors and/or could involve increased breakdown of NO by oxidants [8]. Major pathways of NO release have been associated with caveolae [9]. We previously reported that alterations in the amount of caveolin-1 and caveolin-1/eNOS interaction are involved in aged-related adjustment to hypovolemic state induced by acute hemorrhage in cardiac tissue [10]. This protein also is founded in the vasculature and distal renal epithelia [11]. Caveolin-1 is a negative regulator of endothelial nitric oxide synthase (eNOS), as its interaction and 
binding suppress the activity of eNOS by making caveolin-eNOS complex [12]. In addition, there is evidence to show that caveolin-1 would be a prime candidate for such a role as a major determinant of the aging process [13,14]. Nonetheless, it remains unclear whether caveolin-1-mediated inhibition of activated eNOS plays a major role following hemorrhage and whether it contributes to the effectiveness of compensatory mechanisms to hypovolemic state with aging in the kidney. Therefore, the present study has been designed to investigate: i) NOS activity and protein levels of the different NOS isoforms, ii) the abundance of caveolin-1 and, iii) caveolin-1/eNOS association during hypovolemic state of young and middle-aged rats in the kidney.

\section{Materials and Methods}

The experiments were performed on male Sprague-Dawley rats of ages 2 months (young) and 12 months (middle-aged) years old. The rats were housed in separate cages in a temperaturecontrolled room with a 12:12-h light-dark cycle. They were fed with standard rat chow and tap water ad libitum. Animal care was carried out according to 8th Edition of Guide for the Care and Use of Laboratory Animals published by the National Academy of Sciences, The National Academies Press, Washington DC, United States of America and according to Res. $N^{\circ} 6344 / 96$ of Argentinean National Drug, Food and Medical Technology Administration (ANMAT). Experimental procedures were approved by the ethics committee of the School of Biochemistry and Pharmacy (CEFFB), Buenos Aires University, Argentina.

\section{Animal preparation}

All rats were anesthetized with urethane $(1.0 \mathrm{~g} / \mathrm{kg}$, ip $)$ and kept under anesthesia by additional small doses of urethane throughout the experiment. The rats were placed on a thermostatically controlled heated table. Body temperature was monitored with a rectal thermometer and maintained between 36 and $38^{\circ} \mathrm{C}$. A tracheotomy was performed with use of polyethylene (PE-240) tubing to ensure an open airway. The left femoral artery was cannulated to facilitate blood withdrawal. After an equilibrium period of $15 \mathrm{~min}$ the rats were bled via a catheter using a syringe at a constant rate for $2 \mathrm{~min}$. Hemorrhage ( $20 \%$ of the volemia) was induced by withdrawing blood ( $1.4 \mathrm{ml} / 100 \mathrm{~g}$ body weight). The total amount of blood withdrawn was kept constant (total blood volume was at about $7.0 \pm 0.1 \mathrm{ml} / 100 \mathrm{~g}$ body weight).

\section{Experimental protocols}

Two experimental groups were used for the study of different aged animals:

a. Sham rats (S). After a 15 minutes stabilization period, animals were maintained in basal condition during $120 \mathrm{~min}(\mathrm{n}=14)$.

b. Hemorrhaged rats $(\mathrm{H})$. After a 15 minutes stabilization period, the rats were subjected to arterial hemorrhage of $20 \%$ of the volemia. Thereafter, animals were maintained in experimental condition during $120 \mathrm{~min}(\mathrm{n}=14)$.

\section{Serum creatinine measurement}

From each animal serum samples were collected at time 0 and 120 min of experimental period. Serum creatinine was determined on the day of sample collection and it was measured by a modified Jaffe method with protein precipitation using an alkaline picrate reaction (Wiener Laboratorios S.A.I.C.).

Nitric oxide synthase activity by measuring the conversion of [14C (U)]-L-arginine to [14C (U)]-L-citrulline

NOS activity was determined in renal inner medulla from $\mathrm{S}$ and $\mathrm{H}$ animals after 120 min of beginning the experiment by measuring the conversion of [14C (U)]-L-arginine to [14C (U)]-L-citrulline. Tissue homogenates (approximately $50 \mu$ g protein) were incubated in Tris-HCl buffer ( $\mathrm{pH}$ 7.4) containing $1 \mu \mathrm{g} / \mathrm{mL}$ L-arginine, [14C (U)]-L-arginine (346 $\mu \mathrm{Ci} / \mathrm{mL}), \mathrm{L}$-valine (67 mM), NADPH (1 mM), calmodulin (30 nM), tetrahydrobiopterin $(5 \mu \mathrm{M})$, and $\mathrm{CaCl}_{2}(2 \mathrm{mM})$ for 60 minutes at room temperature. Reaction was stopped by adding $175 \mu \mathrm{L}$ of ice-cold HEPES buffer ( $\mathrm{pH}$ 5.5) containing EDTA (2 $\mathrm{mM}$ ) and then applying to AG 50W cation exchange resin columns. The amount of [14C (U)]-L-citrulline eluted was quantified by liquid scintillation counter (Wallac 1414 WinSpectral; EG\&G Company, Turku, Finland) as described previously. All compounds were purchased from Sigma Chemical Co (Saint Louis, MO, USA). except [U-14C]-L-arginine monohydrochloride $(346 \mathrm{mCi} / \mathrm{mmol}$, Wallac 1414 WinSpectral; EG\&G Company, Turku, Finland). Protein determination was made using dye-binding assay (Bio-Rad, Munchen, Germany) with bovine serum albumin as a standard.

\section{Western blotting analysis}

In order to determine the effect of hypovolemic state on nNOS, eNOS, iNOS and caveolin-1 protein levels, freshly renal inner medulla were isolated from $\mathrm{S}$ and $\mathrm{H}$ groups $(\mathrm{n}=4$ each group at each time). Rats of both groups were sacrificed at $120 \mathrm{~min}$ after the surgery and/or bleeding. The tissue samples were homogenized on ice with a Tissue Tearor (Biospec Products Inc, Bartlesville, OK, USA) in homogenization buffer $(50 \mathrm{mmol} / \mathrm{l}$ Tris, $0.1 \mathrm{mmol} / \mathrm{l}$ EDTA, $0.1 \mathrm{mmol} / \mathrm{l}$ EGTA, 1\% Triton, $1 \mathrm{mmol} / \mathrm{l}$ PMSF, $1 \mu \mathrm{mol} / \mathrm{l}$ pepstatin, $2 \mu \mathrm{mol} / \mathrm{l}$ leupeptin) $1 \mathrm{x}$ protease inhibitor cocktail from Roche Diagnostics. The protein concentration in the Triton-soluble supernatant was determined using the Lowry assay. Equal amounts of protein samples $(45 \mu \mathrm{g})$ were separated in 7.5 \% SDS-PAGE and transferred to a nitrocellulose membrane (Bio-Rad, Munchen, Germany), followed by a primary incubation with rabbit polyclonal anti-NOS antibodies (dilution 1:500: anti-iNOS, epitope at the carboxy terminus; anti-eNOS, epitope at the amino terminus and anti-nNOS, epitope at the amino terminus; BD Biosciences, Franklin Lakes, NJ, USA) or with rabbit polyclonal anti caveolin-1 antibody (dilution 1:500; Santa Cruz Biotechnology, San Diego, CA, USA), 
and a secondary immunoreaction with a goat anti-rabbit antibody conjugated with horseradish peroxidase (dilution 1:5000; Bio Rad, Munchen, Germany). Samples were revealed by chemiluminescence using ECL reagent (Amersham Pharmacia Biotech, Piscataway, NJ, USA) for 2-4 min. The density of bands was quantified by densitometric scanning of Western blots using a Hewlett-Packard scanner and Totallab analyser software (Biodynamics Corp., Seattle, WA, USA) and protein amounts were calculated compared with the densitometric values of the corresponding. Protein levels were expressed as a ratio of the optical densities of the eNOS, iNOS and caveolin-1 bands and the b-actin (BD Biosciences, Franklin Lakes, $\mathrm{NJ}$, USA) band to control for any inaccuracies in the protein loading.

\section{Immunofluorescence}

Coimmunoprecipitation analysis to study the interaction of caveolin-1 with eNOS was performed on renal inner medulla at 120 min after bleeding $(n=4)$. The tissues from $S$ and $H$ group animals were fixed with a solution containing $4 \%$ paraformaldehyde in phosphate-buffered saline (PBS) at $4^{\circ} \mathrm{C}$. The inner medullas were excised, sliced and postfixed overnight in the same fixative solution and washed extensively. Fixed kidney blocks were infiltrated overnight in $30 \%$ sucrose and frozen at $-80^{\circ} \mathrm{C}$. Cryostat sections (thickness: $16 \mu \mathrm{m}$ ) were obtained/performed using a Leitz cryostat and were collected on gelatine subbed glass slides. After washing PBS and fixation in acetone, slides were blocked with 10\% normal goat serum in PBS for 60 min at room temperature. The slices were then incubated with mouse monoclonal anti-eNOS (dilution 1:500, BD Biosciences, Franklin Lakes, NJ, USA) and rabbit polyclonal anticaveolin-1 (dilution 1:500, Santa Cruz Biotechnology, San Diego, CA, USA) at $4^{\circ} \mathrm{C}$ overnight. After washing in PBS, the fluorescent secondary antibodies, rodamine-labelled goat anti rabbit IgG (dilution 1:50, Santa Cruz Biotechnology, San Diego, CA, USA) and fluoresceine-labelled goat anti mousse IgG (dilution 1:50,
Santa Cruz Biotechnology, San Diego, CA, USA), were applied and incubated for $1 \mathrm{~h}$ at room temperature. After washing in PBS, the slides were mounted in a PBS/glycerol (3/1 vol/vol) solution before observation under fluorescence microscope (Olympus BX 51). Control sections that were only incubated with the primary or secondary antibody showed no fluorescence (data not shown). Colocalization was visualized by superimposing the red caveolin-1 over the green eNOS using QCapture Pro 6.0 (QImaging Corporation, 2006) with the resultant yellow image representing the area of colocalization.

\section{Statistical analysis}

Data are mean \pm SEM. The Student's $t$ test paired was used to compare changes in hemodynamic parameters. Analysis of variance of a variable (ANOVA) followed by a posteriori Bonferroni test for multiple comparisons were used for the analysis of other data. We checked the assumptions of normality, homogeneity of variances and independence of the data. The 5\% probability level was used as a criterion for significance. The software Prism (Graph Pad Software, San Diego, CA, USA) was used for statistical analysis.

\section{Results}

\section{Serum creatinine}

In clinical practice, the detection of impaired renal function, which is characterized by a rapid decline of the glomerular filtration rate, is based on an increase of serum creatinine [15]. The hemorrhaged rats and the controls did not statistically differ in respect to serum creatinine in both, young and middle-aged animals (Young animals: S group $0 \mathrm{~min}: 0,72 \pm 0,08 \mathrm{mg} / \mathrm{dl}, 120 \mathrm{~min}$ : $0,81 \pm 0,07 \mathrm{mg} / \mathrm{dl}$; H group $0 \mathrm{~min}: 0,67 \pm 0,07 \mathrm{mg} / \mathrm{dl}, 120 \mathrm{~min}:$ 0,71 $\pm 0,05 \mathrm{mg} / \mathrm{dl}$. Middle-aged animals: $\mathrm{S}$ group $0 \mathrm{~min}: 0,74 \pm 0,09 \mathrm{mg} /$ $\mathrm{dl}, 120 \mathrm{~min}: 0,76 \pm 0,04 \mathrm{mg} / \mathrm{dl} ; \mathrm{H}$ group $0 \mathrm{~min}: 0,65 \pm 0,05 \mathrm{mg} / \mathrm{dl}$, $120 \mathrm{~min}: 0,66 \pm 0,09 \mathrm{mg} / \mathrm{dl} ; \mathrm{p}=\mathrm{NS}$ ).

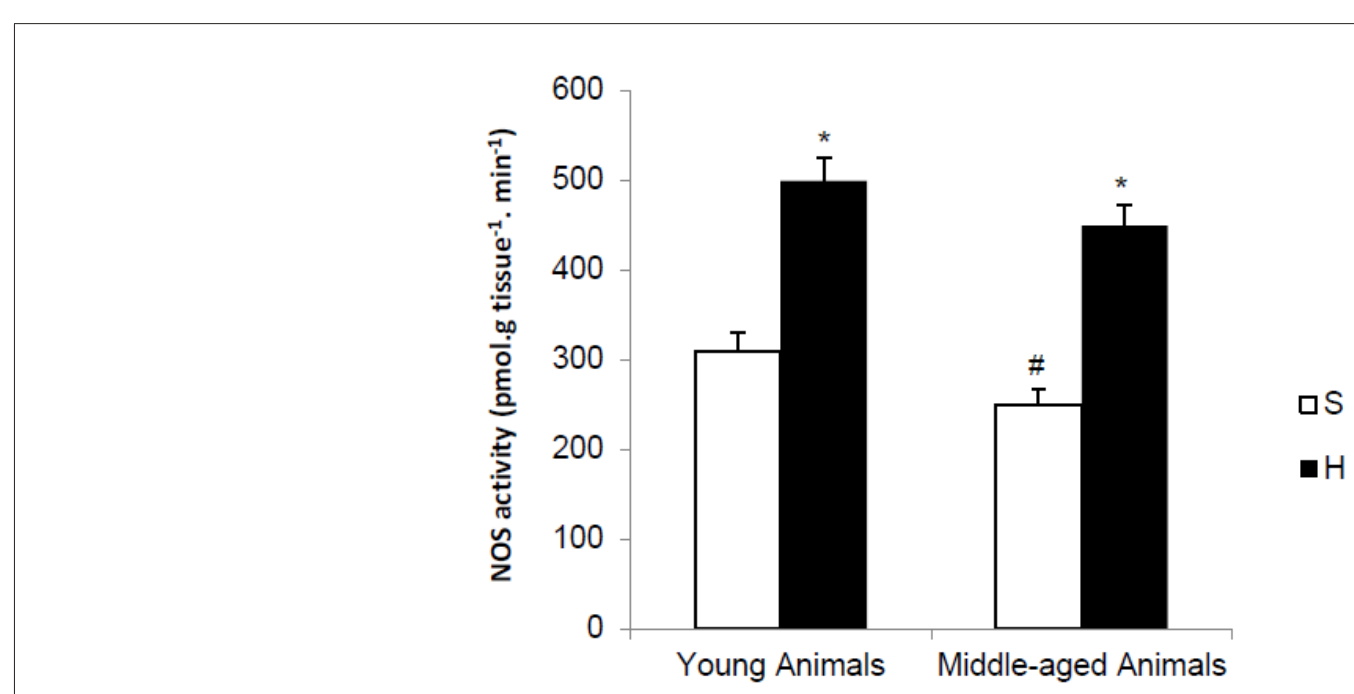

Figure 1: NOS activity in renal inner medulla of young and middle-aged rats subjected to acute hemorrhage. Values are mean $\pm \mathrm{SEM}$. ${ }^{*}<0.01$ vs $\mathrm{S}$ group; \# $\mathrm{P}<0.01$ vs young animals from each group. 


\section{NOS activity}

The potential ability to produce NO by the renal inner medulla was assessed by measuring NOS activity by measuring the conversion of [14C (U)]-L-arginine to [14C (U)]-L-citrulline by in the kidney of young and middle-aged rats, as illustrated in Figure 1. In $\mathrm{S}$ group, kidney NOS activity was significantly smaller in middleaged than in young animals. Hemorrhage increased NOS activity in young and middle-aged animals compared with S group (62\% and $56 \%$, respectively).

\section{Western blot of NOS isoforms and caveolin-1}

To assess the specificity of the expressional regulation of
NOS with hemorrhage and aging, we analyzed the variations of the abundance of NOS isoforms in the renal inner medulla of all groups of animals. In assessing the protein levels of the different isoforms of NOS in the renal medulla is observed that the advance of age and acute blood volume loss of $20 \%$ not cause changes from baseline values of the S Groups. Because the activity of eNOS was shown to be regulated posttranslationally through its inhibitory interaction with the caveolar coat protein caveolin, we assessed potential changes in the abundance of the caveolin-1 expressed in renal medulla by Western blot analysis. The results showed that the hypovolemic state and aging are two factors that do not change the levels of this protein in the renal medulla (Figure 2.).

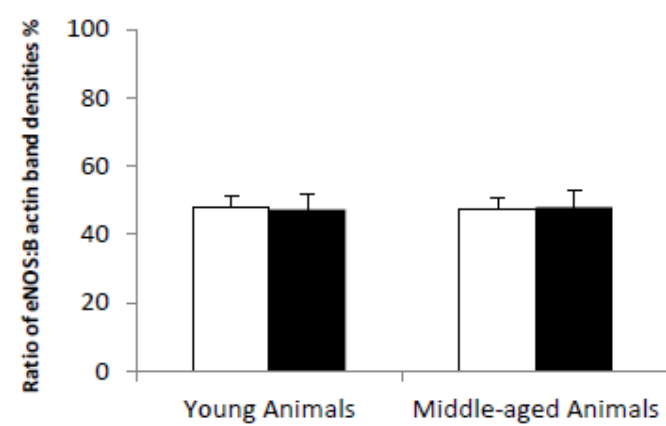

eNOS

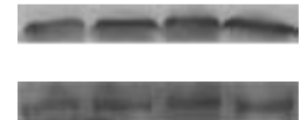

c

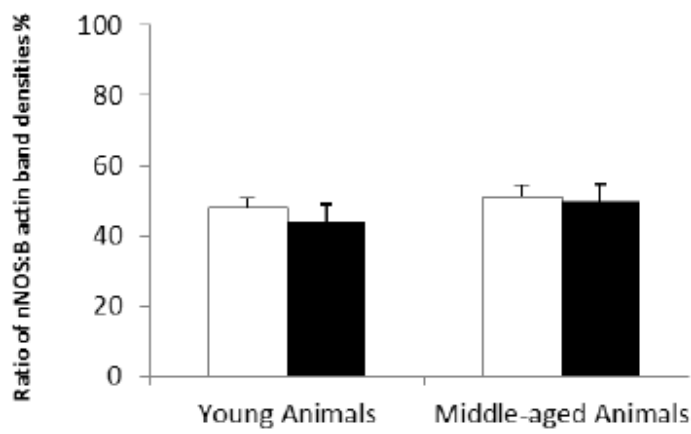

$\mathrm{nNOS}$

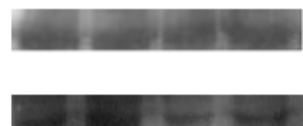

B

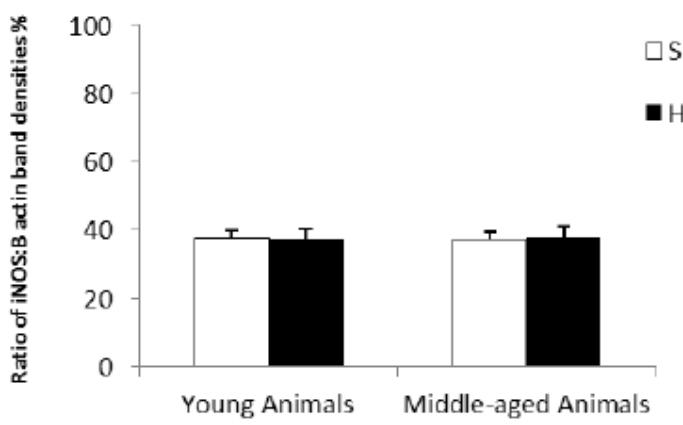

iNOS

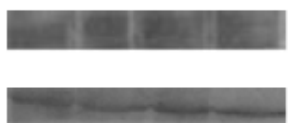

D

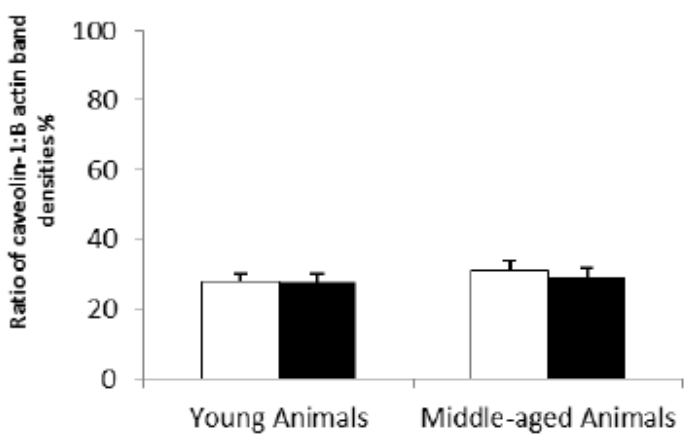

Caveolin-1

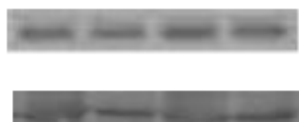

Figure 2: Representative Western blots of eNOS, iNOS, nNOS and caveolin-1 carried out on proteins from renal inner medulla of young and middle-aged rats subjected to acute hemorrhage. Histograms illustrate the mean eNOS, iNOS, nNOS and caveolin-1 protein values for each group. Data are mean $\pm \mathrm{SEM}$. ${ }^{*} \mathrm{P}<0.05$ vs $\mathrm{S}$ group; \#P $<0.05$ vs young animals from each group. $\beta$-actin served as an internal control. 


\section{Immunoprecipitation assay for e-NOS and caveolin-1} interaction

To assess the potential for the association of eNOS with the inhibitory protein caveolin-1 in situ, eNOS was immunoprecipitated with anti-caveolin-1 antibodies from whole renal inner medulla extracts of young and middle-aged rats. The presence of caveolin-1 and eNOS in renal tubular cells showed in Figure 3. The study showed a pattern of colocalization of eNOS localization and caveolin- 1 with an increased association of these proteins in middle-aged animals compared to young one in S Group. In H Group, after 120 min of hemorrhage, it sees dissociation between caveolin-1 and eNOS in respect of S Group. This effect is evident in both, young and middleaged animals.

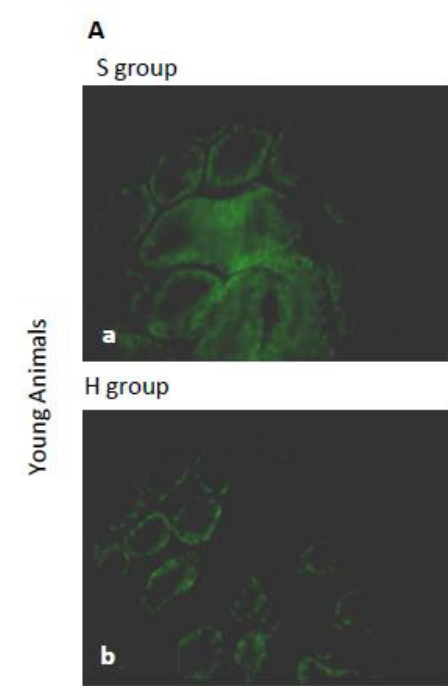

eNOS stain

B

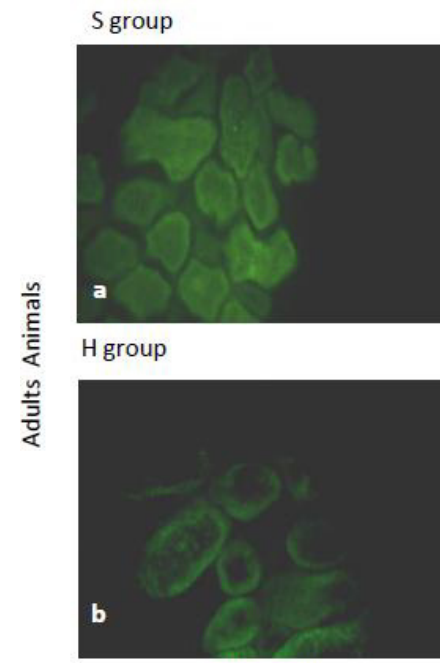

eNOS stain
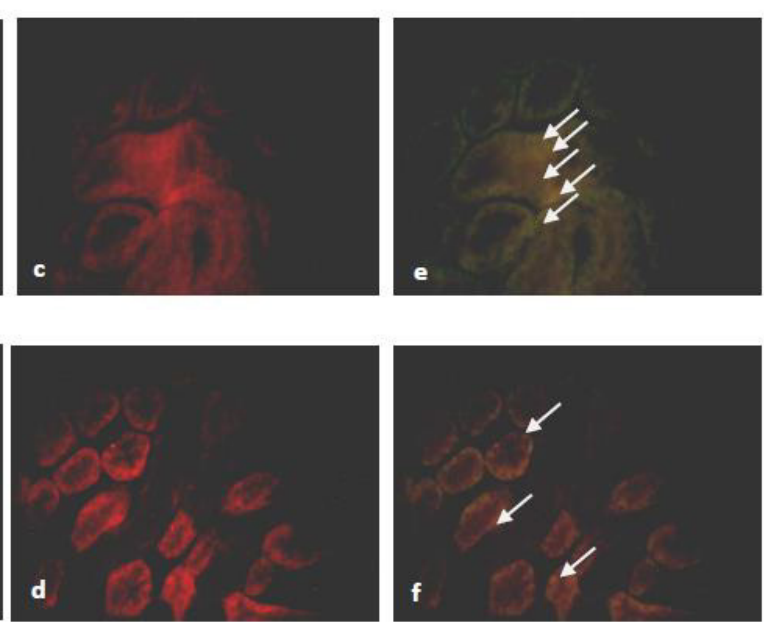

caveolin-1 stain

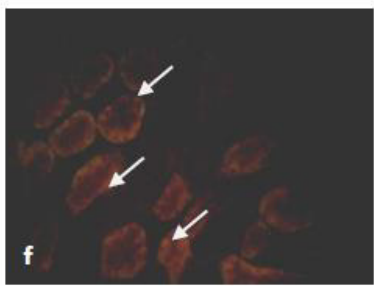

Merge
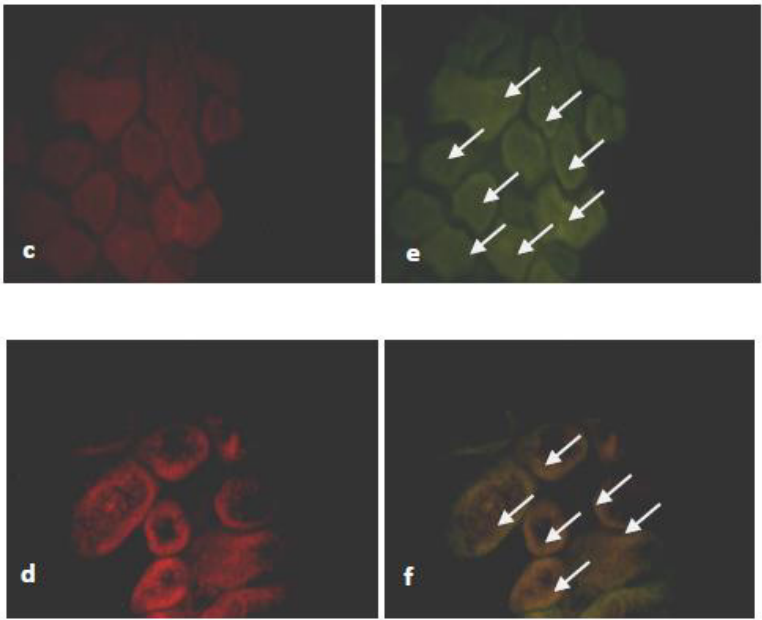

caveolin-1 stain

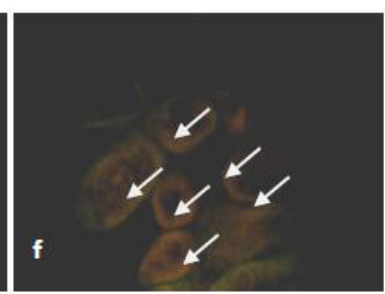

Merge

Figure 3: Caveolin-1 and eNOS distributions in renal inner medulla of young and middle-aged rats subjected to acute hemorrhage. Rat kidney sections were double immunolabeled with anti-eNOS monoclonal antibody (a-b) and anti-caveolin-1 polyclonal antibody (c-d). Merged images are shown in e-f. The main areas of colocalization are indicated by white arrows. (Bar $=20 \mu \mathrm{m})$.

\section{Discussion}

Our results provide further support new evidence that caveolin-1 is involved in the regulation of renal NOS activity in response to acute hemorrhage and aging. The advancement of the current study is that association/dissociation of caveolin-1 and eNOS in renal medulla play a critical role in preserving the renal function during hypovolemic state in young and middle-aged rats. NO plays a pivotal role in regulation of renal hemodynamics by different NOS isoforms in the kidney [16-19]. In addition, studies in vivo and in vitro show that NO pathway is one of several modulators involved in the aging process [20,21]. The present study revealed a significant reduction of renal medulla NOS activity 
but no significant change in the abundance of NOS isoforms in middle-aged rats compared with the corresponding values in the young animals. These results are consistent with those of Erdely et al. [8] who reported that renal NO synthase activity is decreased with advancing age. Moreover, it was reported that loss of NO availability in the aged kidney (16 and 24 months old) would be expected to result in several adverse effects, including decreased renal perfusion, increased renal vasoconstriction, and enhancement of fibrosis [22]. Different circulating endocrine and local paracrine factors such as NO have been postulated to modulate the hemodynamic responses to hypovolemia [23]. There is good evidence that NO overproduction importantly contributes to vascular hyporeactivity and vascular decompensation following acute hemorrhage $[24,25]$. On the other hand, a small amount of NO is pivotal for the preservation of microvascular perfusion [26]. We previously demonstrated that hypovolemic state induced by acute hemorrhage (20\% of the volemia) triggered a heterogeneous and dynamic NOS activation modulating the cardiovascular response [27]. We report here that hypovolemic state led to upregulation of renal medulla NOS activity in both, young and middle-aged rats. Our findings support the hypothesis that acute hemorrhage induces an increase of availability of renal NO, which targets a pivotal protective function by maintaining renal homeostasis against organ injury and dysfunction associated with hypovolemic state. It is proved that NOS activity was affected by its gene expression and (reversible) post-translational modification of the enzyme, such as methylation of the eNOS promoter, NOS phosphorylation, and protein-protein interactions and so on [28]. Studies have suggested that caveolin-1 was expressed and localized in endothelial cells of arteries, arterioles, and glomeruli. Caveolin-1 immunoreactivity was also apparent in the vascular smooth muscle cells of glomerular arterioles and in basolateral aspects of distal tubules [28]. Binding of eNOS to caveolin-1 on plasma membrane inhibits the activity of this NOS isoform. However, caveolin-1 has no effect on cytosolic NOS isoforms (iNOS, nNOS) [29]. Since caveolin-1 play a central role in regulation of NO production by eNOS, we sought to examine the effect of acute hemorrhage and aging on abundance of this protein in the kidney. Our present results showed that the abundance of caveolin-1did not change after 120 min of bleeding or with aging. However, in addition to the absolute changes in the abundance of caveolin, it is also significant to consider whether the change in the tissue and subcellular distribution of this protein may influence the activity of eNOS. This issue is of potential importance, because it could explain the maintaining physiological regulation of renal function during hypovolemic state and age by NO unexpected from unchanged abundance of eNOS (or other NOS isoforms). Consistently, we found that aging induces a significant increase of colocalization of eNOS with caveolin-1, suggesting that this association limits renal NOS activity under physiological conditions in response to aging. There have been several reports that organs, such as brain and spleen, expressed caveolin-1 protein in significantly higher amounts only in the aged state [30]. In addition, the up-regulation of caveolin with aging is suggested to relate to insulin resistance, resulting in susceptibility to diabetes mellitus [31]. In this work, we have demonstrated that caveolin - 1 would play an important role in the regulation of renal NOS activity in the middle-aged rats. The organ-specific regulation of caveolins in an age-dependent manner would explain the characteristic aging process of the respective organ. Although the precise mechanisms by which acute hemorrhage induces an increase of NOS activity in the kidney are not fully clear, the decreased in eNOS/ caveolin-1 association might be a major factor. Immunoprecipitation experiments showed that acute hemorrhage decreased the association of caveolin-1 and eNOS suggesting that acute hemorrhage could activate eNOS in renal medulla in both, young and middle-aged animals. To our knowledge, this is novel study to provide direct evidence to demonstrate the relationship between increased NOS activity and dissociation of eNOS from caveolin-1 in the kidney and their link to hypovolemic state induced by acute bleeding during aging process.

\section{Conclusion}

Our findings further highlight the pivotal role of renal caveolin-1/eNOS/NO pathway to preserve renal alterations triggered by hypovolemic state during chronologic advance of age. This observation would be consistent with data suggesting the dependence of renal hemodynamics on the NO bioavailability $[32,33]$. The underlying molecular mechanisms are yet to be elucidated; therefore, further studies are required in the future.

\section{Conflict of Interest}

None.

\section{Acknowledgment}

This work was supported by the Universidad de Buenos Aires grants B022.

\section{References}

1. Eriksson M, Brattstrom O, Martensson J, , Emma L, Oldner A (2015) Acute kidney injury following severe trauma: risk factors and long-term outcome. J Trauma Acute Care Surg 79(3): 407-412.

2. Herrera M, Garvin JL (2005) Recent Advances in the Regulation of Nitric Oxide in the Kidney. Hypertension 45(6): 1062-1067.

3. Koneru S, Penumathsa SV, Thirunavukkarasu M, Samuel SM, Zhan L, et al. (2007) Redox regulation of ischemic preconditioning is mediated by the differential activation of caveolins and their association with eNOS and glut-4. Am J Physiol Heart Cir Physiol 292(5): H2060-H2072.

4. Willière Y, Borschewski A, Patzak A, Nikitina T, Dittmayer C, et al. (2018) Caveolin 1 Promotes Renal Water and Salt Reabsorption. Scientific reports 8(1): 545 .

5. Anderson S, Eldadah B, Halter JB, Hazzard WR, Himmelfarb J, et al. (2011) Acute Kidney Injury in Older Adults. J Am Soc Nephrol 22(1): 28-38. 
6. Regel G, Gotz M, Weltner T, Sturm JA, Tscherne H (1996) Pattern of organ failure following severe trauma. Wored J Surg 20(4): 422-429.

7. Morgan WM, O'Neill JA (1998) Hemorrhagic and obstructive shock in pediatric patients. New Horiz 6(2): 150-154.

8. Erdely A, Greenfeld Z, Wagner L (2003) Sexual dimorphism in the aging kidney: Effects on injury and nitric oxide system. Kidney Int 63(3): 1021-1026.

9. Fridolfsson HN, Roth DM, Insel PA (2014) Regulation of intracellular signaling and function by caveolin. FASEB J Off Publ Fed Am Soc Exp Biol 28(9): 3823-3831.

10. Arreche ND, Sarati LI, Martinez CR, Andrea LF, Balaszczuk AM (2012) Contribution of caveolin-1 to ventricular nitric oxide in age-related adaptation to hypovolemic state. Regulatory Peptides 179(1-3): 43-49.

11. Xin Feng, Wei Gao, Yao Li (2017) Caveolin-1 is involved in high glucose accelerated human glomerular mesangial cell senescence. Korean J Intern Med 32(5): 883-889.

12. Minshall RD, Tiruppathi C, Vogels SM (2002) Vesicle formation and traffickng in endothelial cells and regulation of endothelial barrier function. Histochem Cell Biol 117(2): 105-112.

13. Cho KA, Park SC (2005) Caveolin-1 as a prime modulator of aging: a new modality for phenotypic restoration? Mech Ageing Dev 126(1): 105-110.

14. Nguyen K, Cho K (2017) Versatile functions of caveolin-1 in agingrelated diseases. Chonnam medical journal 53(1): 28-36.

15. Kellum JA, Levin N, Bouman C (2002) Developing a consensus classification for acute renal failure. Curr Opin Crit Care 8(6): 509-514.

16. Guan Z, Gobe G, Willgoss D (2006) Renal endothelial dysfunction and impaired autoregulation after ischemia-reperfusion injury result from excess nitric oxide. Am J Physiol Renal Physiol 291: F619-F628.

17. Singh I, Grams M, Wang WH, Yang T, Killen P, et al. (1996) Coordinate regulation of renal expression of nitric oxide synthase, rennin, and angiotensinogen mRNA by dietary salt. Am J Physiol 270 (6 Pt 2): F10271037.

18. Tolins JP, Shultz PJ (1994) Endogenous nitric oxide synthesis determines sensitivity to the pressure effect of salt. Kidney Int 46(1): 230-236.

19. Ying WZ, Sanders PW (1998) Dietary salt enhances glomerular endothelial nitric oxide synthase through TGF B1. Am J Physiol 275(1): F18-F24.

20. Vasa M, Breitschopf K, Zeiher AM (2000) Nitric oxide activates telomerase and delays endothelial cell senescence. Circ Res 87(7): 540-542.

21. Hoffmann J, Haendeler J, Aicher A, Rössig L, Vasa M, et al. Aging enhances the sensitivity of endothelial cells toward apoptotic stimuli: important role of nitric oxide. Circ Res 89(8): 709-715.
22. Satoh M, Kidokoro K, Ozeki M, Nagasu H, Nishi Y, et al. (2013) Angiostatin production increases in response to decreased nitric oxide in aging rat kidney. Laboratory Investigation 93(3): 334-343.

23. Cabrales P, Tsai AG, Intaglietta M (2009) Exogenous nitric oxide induces protection during hemorrhagic shock. Resuscitation 80(6): 707-712.

24. Liu L, Ward JA, Dubick MA (2003) Hemorrhage-Induced Vascular Hyporeactivity to Norepinephrine in Select Vasculatures of Rats and the Roles of Nitric Oxide and Endothelin. Shock 19(3): 208-214.

25. Huang HC, Chen YC, Wang SS, Che-Chang C, Fa-Yauh L, et al. (2004) Nitric oxide synthase expression in the splanchnic hyposensitivity to glypressin of a hemorrhage-transfused rat model with portal hypertension. J Chin Med Assoc 67(6): 268-274.

26. Wang Y, Yan J, Xi L, Qian Z, Wang Z, et al. (2012) Protective effect of crocetin on hemorrhagic shock induced acute renal failure in rats. Shock 38(1): 63-67.

27. Balaszczuk AM, Arreche ND, Mc Laughlin M, Arranz C, Fellet AL, et al. (2006) Nitric oxide synthases are involved in the modulation of cardiovascular adaptation in hemorrhaged rats. Vascular Pharmacology 44(6): 417-426.

28. Peng XL, Qu W, Wang LZ, Huang BQ, Ying CJ, et al. (2014) Resveratrol ameliorates high glucose and high-fat/sucrose diet-induced vascular hyperpermeability involving Cav-1/eNOS regulation. PLoS One 9(11): e113716.

29. Komers R, Schutzer WE, Reed JF, Lindsley JN, Oyama TT, et al. (2006) Altered endothelial nitric oxide synthase targeting and conformation and caveolin-1 expression in the diabetic kidney. Diabetes 55(6): 16511659.

30. Bai Y, Ye S, Mortazavi R (2007) Effect of renal injury-induced neurogenic hypertension on NO synthase, caveolin-1, AKt, calmodulin and soluble guanylate cyclase expressions in the kidney. Am J Physiol Renal Physiol 292(3): F974-F980.

31. Park WY, Park JS, Cho KA, Kim DI, Ko YG, et al. (2000) Up-regulation of caveolin attenuates epidermal growth factor signaling in senescent cells. J Biol Chem 275(27): 20847-20852.

32. Muñoz P, Mora S, Sevilla L, Kaliman P, Tomàs E, et al. (1996) Expression and Insulin-regulated Distribution of Caveolin in Skeletal Muscle. The Journal of Biological Chemistry 271(14): 8133-8139.

33. Patterson ME, Mouton CR, Mullins JJ (2005) Interactive effects of superoxide anion and nitric oxide on blood pressure and renal hemodynamics in transgenic rats with inducible malignant hypertension. Am J Physiol Renal Physiol 289(4): F754-F759. 